\title{
The Clinical Value of Myocardial Blood Flow Measurement*
}

\author{
Paolo G. Camici and Ornella E. Rimoldi \\ Medical Research Council, Clinical Sciences Centre and National Heart and Lung Institute, Imperial College, \\ London, United Kingdom
}

\begin{abstract}
PET provides robust and reproducible measurements of regional myocardial blood flow in milliliters per minute per gram of tissue, providing unique pathophysiologic and diagnostic information on the function of the coronary macro- and microcirculation. There is compelling evidence to suggest that in many instances abnormalities of global myocardial perfusion are demonstrated in individuals with either coronary risk factors for coronary artery disease or different myocardial diseases in the absence of angiographically demonstrable stenosis of the epicardial coronary arteries. In this context, measurement of myocardial blood flow gives unique diagnostic information regarding the function of the coronary microcirculation and provides a quantitative surrogate endpoint against which the efficacy of treatments can be established.
\end{abstract}

Key Words: noninvasive measurement; cardiovascular disease; myocardial blood flow

J Nucl Med 2009; 50:1076-1087

DOI: 10.2967/jnumed.108.054478

D espite substantial advances in the diagnosis and treatment of cardiovascular diseases, these diseases remain the main cause of death in our society and have a major impact on public health expenditure. According to the recent report from the American Heart Association Statistics Committee and Stroke Statistics Subcommittee, an estimated 80,700,000 American adults (1 in 3) have one or more types of cardiovascular disease, with a mortality rate in 2004 of 869,724, and more than half of these subjects are younger than $60 \mathrm{y}(1)$. Cardiovascular disease claims approximately as many lives each year as do cancer, chronic lower respiratory disease, accidents, and diabetes mellitus combined. Nearly $70 \%$ of the mortality for

Received Oct. 16, 2008; revision accepted Dec. 29, 2008.

For correspondence or reprints contact: Paolo G. Camici, Medical Research Council, Clinical Sciences Centre, Hammersmith Hospital, Du

Cane Rd., London, W12 ONN United Kingdom.

E-mail: paolo.camici@csc.mrc.ac.uk

*NOTE: FOR CE CREDIT, YOU CAN ACCESS THIS ACTIVITY

THROUGH THE SNM WEB SITE (http://www.snm.org/ce_online) THROUGH JULY 2010.

Paolo G. Camici has indicated he is a consultant/advisor for GE Healthcare. No other potential conflict of interest relevant to this article was reported.

COPYRIGHT @ 2009 by the Society of Nuclear Medicine, Inc. cardiovascular disease is due to coronary heart disease. The direct and indirect costs estimated for 2008 in the United States for cardiovascular disease and stroke are in the range of $\$ 448.5$ billion, with coronary heart disease accounting for $\$ 156.4$ billion, placing cardiovascular diseases at the highest level in comparison with any other diagnostic group (1).

The widespread use of thrombolytic therapy and percutaneous coronary interventions, in association with increasingly potent antithrombotic agents, has contributed to a significant reduction in the mortality and morbidity in patients with acute coronary syndromes. A downside to this success has been the greater number of patients with residual left ventricular (LV) dysfunction undergoing progressive LV remodeling and experiencing congestive heart failure (2). Moreover, the structural changes inducing remodeling of the left ventricle cause an impairment of the microvascular function, which also involves the nonischemic territory (3).

A further significant reduction in the morbidity and mortality from ischemic heart disease can be achieved through more aggressive prevention strategies that would require a better characterization of subtle functional abnormalities of the coronary circulation in the preclinical stages of the disease.

\section{CORONARY MACRO- AND MICROVASCULAR DISEASE}

Atherosclerotic disease of the vascular system is a continuum. Disease may begin early in life, but it does not become clinically overt until atherosclerotic plaques reach a critical stage (Fig. 1).

Noninvasive imaging techniques can be used to diagnose coronary macro- and microvascular disease. The former, which affects the large epicardial arteries, may be symptomatic and is a consequence of the critical growth of atherosclerotic plaques inside the lumen of the vessel. In addition to symptoms, its diagnosis is based either on the identification of the anatomic changes (stenoses) on the large epicardial coronary arteries or on the functional consequences of these stenotic lesions on coronary flow reserve (CFR) or regional LV function. The anatomy of the 
A

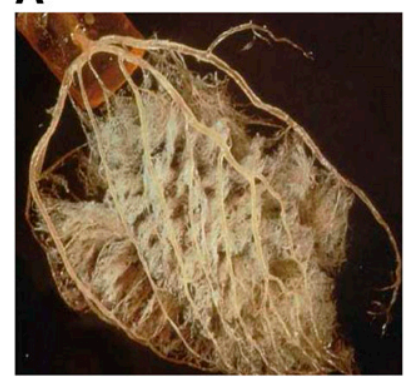

B

FIGURE 1. Coronary macro- and microvascular disease. (A) Postmortem cast of coronary circulation (courtesy of Dr. Michael Gibson) illustrating abundance of microvascular network, which is not visible at coronary angiography (B). Because of its limited spatial resolution, coronary angiography can be used only to study anatomy of large epicardial coronary arteries.

epicardial coronary arteries can be assessed using either invasive angiography or, more recently, multislice CT angiography. Semiquantitative assessment of regional myocardial perfusion with SPECT is a noninvasive, robust, and widely available method of assessing myocardial ischemia and has an obvious role in the clinical setting. Many studies have assessed the sensitivity and specificity of this technique for the detection of coronary artery disease (CAD), coronary arteriography usually being the standard by which the accuracy of scintigraphy is judged (4). Alternatively, changes in regional wall motion during stress can be assessed using echocardiography and cardiovascular magnetic resonance (CMR).

When lesions on the epicardial coronary arteries are subcritical (hemodynamically nonsignificant [i.e., with no obvious effects on tissue perfusion]), they may not manifest themselves until the plaque becomes unstable and ruptures, leading to an acute coronary syndrome. Recent studies have provided preliminary evidence that it is possible to study the anatomy and composition or the functional status of the plaques to identify those at higher risk of rupture. Plaque composition can be imaged with high-resolution CMR, and the inflammatory activity of the plaques can be assessed by means of PET using specific tracers for the detection of inflammation. More studies, however, will be necessary to test the applicability of these techniques in clinical practice.

Another form of early (preclinical) disease is that affecting the coronary microcirculation (small vessels $<300$ $400 \mu \mathrm{m}$ ), which may occur in patients with normal coronary angiograms. Because there is no technique enabling the direct visualization of the coronary microcirculation in vivo, its assessment relies on the measurement of parameters that reflect its functional status, such as absolute myocardial blood flow (MBF) and CFR. A bulk of studies, mainly using PET for the noninvasive quantification of regional $\mathrm{MBF}$, have demonstrated that dysfunction of the coronary microcirculation occurs in many clinical conditions in the absence of demonstrable stenoses on the large epicardial arteries. Studies in subjects who are asymptomatic but have risk factors for CAD such as hypercholesterolemia, essential hypertension, diabetes mellitus, and smoking have provided evidence of how these risk factors translate into measurable damage to the coronary microcirculation in the absence of demonstrable stenoses of the epicardial arteries. In some cases, these abnormalities represent mere epiphenomena, whereas in others they represent important markers of risk or even contribute to the pathogenesis of myocardial ischemia, thus becoming therapeutic targets $(5,6)$.

In a previously published review article, we have discussed in-depth the methodologic aspect of MBF measurement with PET and its main clinical application (7). The scope of the present review is to expand and update this topic by reviewing the literature published since 2005 .

\section{FUNCTIONAL ANATOMY OF CORONARY CIRCULATION AND ISCHEMIC HEART DISEASE}

Understanding the anatomy and physiology of the coronary circulation is the prerequisite for understanding the mechanisms that lead to its derangement. The coronary arterial system is composed of 3 compartments with different functions, although their precise borders cannot be clearly defined anatomically or histologically (Fig. 2).

The proximal compartment is represented by the large epicardial coronary arteries, which have a capacitance function and offer little resistance to coronary blood flow. During systole, epicardial coronary arteries accumulate elastic energy as they increase their blood content up to about $25 \%$. This elastic energy is converted into blood kinetic energy at the beginning of diastole and contributes to the prompt reopening of intramyocardial vessels that are squeezed closed by systole.

The intermediate compartment is represented by prearterioles, which are characterized by a measurable pressure drop along their length. Prearterioles are not under direct vasomotor control by diffusible myocardial metabolites because of their extramyocardial position or arterial wall thickness. Proximal prearterioles are more responsive to changes in flow, whereas distal prearterioles are more responsive to changes in pressure. The distal compartment is represented by arterioles. Because their tone is influenced by myocardial metabolism, arterioles are characterized by a considerable pressure drop along their length and represent the site of the metabolic regulation of MBF. The specific function of arterioles is the matching of myocardial blood supply and myocardial oxygen demand.

Large epicardial arteries have a diameter ranging from a few millimeters to approximately $500 \mu \mathrm{m}$ and are visible at coronary angiography. Prearterioles (diameter, from $\sim 500$ to $\sim 100 \mu \mathrm{m}$ ) and arterioles (diameter $<100 \mu \mathrm{m}$ ) are below the resolution of current angiographic systems and hence are not visible at angiography. Notably, each compartment is governed by distinct regulatory mechanisms $(8,9)$. 


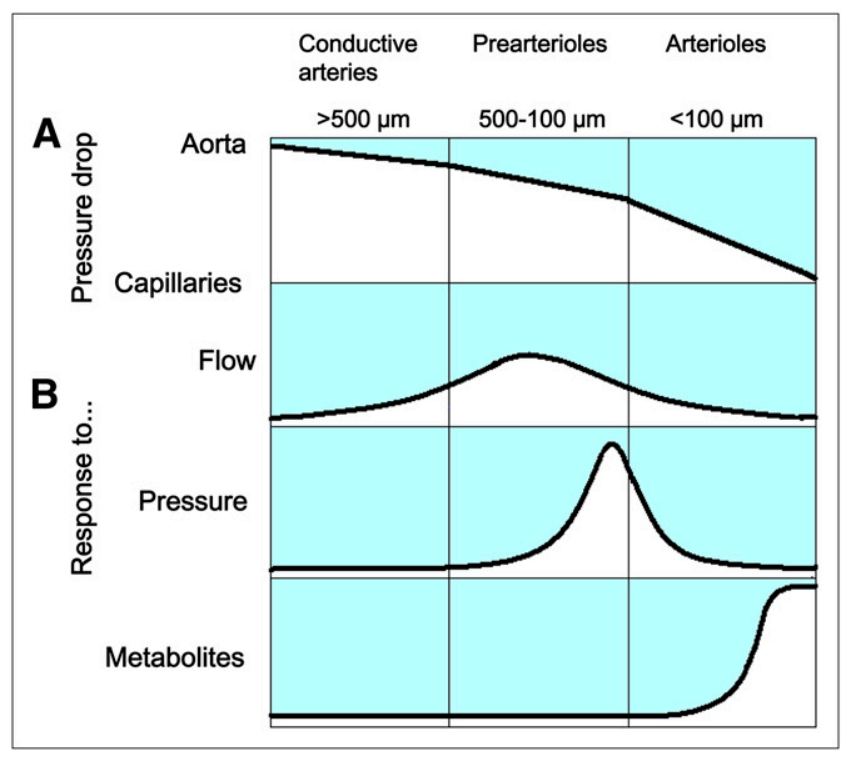

FIGURE 2. Schematic representation of functional subdivision of coronary arterial system in conductive vessels, prearterioles, and arterioles. (A) Pressure drop along conductive vessels is negligible, that through prearterioles is appreciable, and that through arterioles is largest. (B) Conductive arteries and, even more, proximal prearterioles are more responsive to flow-dependent dilatation. Distal prearterioles are more responsive to changes in intravascular pressure and are mainly responsible for autoregulation of coronary blood flow. Arterioles are more responsive to changes in intramyocardial concentration of metabolites and are mainly responsible for metabolic regulation of coronary blood flow. (Adapted from (5).)

The vascular resistance is distributed in series along the coronary vascular bed but also varies in parallel vascular segments in different layers of the ventricular wall.

The individual contribution of successive coronary vascular segments to total resistance can be inferred from the progressive drop in mean pressure from the aorta to the coronary sinus. About $10 \%$ of the pressure drop occurs in epicardial coronary arteries, $30 \%$ in prearterioles, $40 \%$ in arterioles, and $20 \%$ from capillaries to large veins (Fig. 2) (10).

The distribution of coronary resistance in parallel vascular segments in different layers of the ventricular wall has been studied by pressure or flow curves, which provide information on the differences in vascular resistance between subendocardium and subepicardium. Tachycardia impairs subendocardial blood flow, particularly in the presence of myocardial hypertrophy. In fact, the time constant of the diastolic filling of intramyocardial vessels does not shorten with heart rate and becomes a limiting factor for the perfusion of subendocardial layers. Nevertheless, in conscious dogs, under normal resting conditions, the subendocardial flow is higher than the subepicardial flow (with a ratio of about 1.25:1), due to a greater conductance of subendocardial arterioles. This latter find- ing is consistent with the higher subendocardial oxygen demand, which is secondary to the greater wall stress in subendocardial than in subepicardial layers (11). Thus, given a sufficiently high perfusion pressure, a sufficiently long diastole, and an adequate systolic expansion of conductive arteries, the subendocardium is adequately perfused. However, when perfusion pressure at the origin of the arteriolar vessels, compared with that of the aorta, is reduced, perfusion becomes jeopardized earlier in the subendocardial than in the subepicardial layers of the left ventricle. The selective constriction of subepicardial vessels can influence perfusion pressure in subendocardial vessels and hence subendocardial flow (11). In addition, the subendocardium becomes even more susceptible to underperfusion if diastolic time is short and myocardial hypertrophy is present $(12,13)$.

\section{Response of Coronary Circulation to Changes in Flow}

The shear stress is proportional to the shear rate or velocity and to viscosity. Arteries exhibit an intrinsic tendency to maintain a shear stress constant, despite changes in shear rate or in viscosity. Indeed, high or low shear stress may jeopardize the interaction between blood elements and endothelium. In the absence of changes in distending pressure, variations of flow in epicardial coronary arteries can be achieved by intracoronary injection of arteriolar vasodilators (14). Flow-mediated dilatation occurs also in proximal prearterioles during dilatation of distal prearterioles in response to a reduction in perfusion pressure or increased myocardial oxygen consumption or after myocardial ischemia.

Flow-mediated dilatation is achieved through the release by endothelial cells of vasodilators in response to an increase in shear stress, in particular nitric oxide (NO), endotheliumderived hyperpolarizing factor (EDHF), and prostacyclin. NO appears to play a key role in flow-mediated relaxation of large epicardial vessels, as relaxation is prevented by $\mathrm{N}^{\mathrm{G}_{-}}$ monomethyl-L-arginine (L-NMMA), a specific inhibitor of NO synthesis. The contribution of EDHF to endotheliumdependent relaxation varies as a function of the size of the artery; indeed, it is more pronounced in resistance vessels and might play an important role in prearteriolar flow-mediated dilatation. In contrast, the contribution of prostacyclin to flow-mediated relaxation appears to be modest. Nevertheless, prostacyclin-mediated relaxation might be important in the presence of endothelial dysfunction with reduced bioavailability of $\mathrm{NO}$, when it may provide a compensatory mechanism (15).

Coronary autoregulation is the intrinsic ability to maintain a constant blood flow in the face of changes in perfusion pressure. Variations of coronary perfusion pressure in the beating heart-in the presence of unaltered myocardial metabolic requirements - can be achieved by perfusing the coronary circulation independently from the aorta, so that aortic pressure remains constant when coronary arterial pressure is varied. In this setting, pressure or 
flow curves show that when perfusion pressure is varied, flow remains nearly constant over a wide range of pressures (i.e., from $\sim 60$ to $\sim 120 \mathrm{~mm} \mathrm{Hg}$ ). The level at which flow remains constant is determined by the levels of myocardial oxygen consumption; when the latter is low the plateau of flow is low, and when oxygen consumption is high the plateau is high. Notably, for decreasing perfusion pressures, autoregulation is better maintained in the subepicardium than in the subendocardium, which, therefore, is more susceptible to the detrimental effects of low perfusion pressures.

The mechanism responsible for autoregulation is probably a myogenic response of distal prearteriolar vessels: They vary their diameter as a function of perfusion pressure (16).

Under basal resting conditions, the tone of the coronary resistive vessels is high. This intrinsically high resting tone allows the coronary circulation to increase flow when myocardial oxygen consumption increases through rapid changes in small vessel diameter, a mechanism known as functional hyperemia. This metabolic control of coronary blood flow is precise and fundamental for adequate myocardial oxygen supply and it results from the balance of the action of neurotransmitters, autoacoids produced by endothelial cells (NO, prostaglandins, EDHF, endothelin), adventitial cells (histamine, kinins, leukotrienes, blood-borne substances [thromboxane $\mathrm{A}_{2}$, serotonin]), and vasoactive drugs. When myocardial oxygen consumption remains constant, any change in flow caused by neurohumoral modulation is mirrored by a change in oxygen extraction, so that vasodilatation or constriction are associated, respectively, with a proportional increase or decrease in coronary sinus $\mathrm{PO}_{2}$ and oxygen saturation.

By acting on coronary arterioles, adenosine is one of the key mediators of metabolic blood flow regulation. Adenosine is formed by degradation of adenine nucleotides under conditions in which adenosine triphosphate use exceeds the capacity of myocardial cells to resynthesize high-energy compounds, resulting in the production of adenosine monophosphate (which is converted to adenosine by the enzyme 5 '-nucleotidase). Adenosine then diffuses from myocytes into interstitial fluid, where it exerts powerful arteriolar dilator effects through the stimulation of $\mathrm{A}_{2}$ adenosine receptors on smooth muscle cells. Adenosine production increases in the case of an imbalance in the supply or demand ratio of myocardial oxygen, with the rise in interstitial concentration of adenosine paralleling the increase in coronary blood flow $(17,18)$.

Changes in myocardial oxygen consumption trigger a complex and integrated microvascular response. The arterioles dilate in response to the release of myocardial metabolites, and this dilatation decreases both resistance in the overall network and pressure in distal prearterioles, which induces myogenically sensitive vessels to dilate. In turn, dilatation of distal prearterioles and arterioles results in an increase in shear stress and triggers flow-dependent dilatation in larger prearterioles and in conductance arteries.
Thus, as proposed by Chilian, the coronary circulation matches oxygen requirements with blood flow by coordinating the resistances within different microvascular domains, each controlled by distinct and integrated regulatory mechanisms. This integration, and the apparent redundancy of control mechanisms, has the advantage of compensating for dysfunctions ensuing in one mechanism to maintain an adequate level of perfusion $(9,16)$.

\section{Response of Coronary Circulation to Ischemia}

Reactive hyperemia occurs within a few seconds on release of a short occlusion of a conduit coronary artery; the peak flow reaches 4-5 times the value of preischemic flow and is directly correlated to the duration of the ischemic period for occlusion times up to $15-20 \mathrm{~s}$. Although longer occlusions do not modify further the peak of the hyperemic response, they do affect the duration of the entire hyperemic process, which increases proportionally with the length of the occlusion.

It is generally accepted that myocardial ischemia, even that lasting only briefly, is the most effective stimulus for vasodilatation of coronary resistive vessels and that, under normal circumstances, reactive hyperemic peak flow represents the maximum flow available at a given coronary perfusion pressure. Values of coronary blood flow comparable to the peak flow of reactive hyperemia can be achieved using coronary vasodilators such as intravenous adenosine or dipyridamole, which induce a near-maximal vasodilatation of the coronary microcirculation $(14,19)$. CFR is the ratio of maximum (hyperemic) coronary or $\mathrm{MBF}$ to resting basal coronary or MBF. In the absence of coronary stenoses, a reduced CFR reflects dysfunction of the coronary microcirculation. This characterization requires quantitative measurements of pressure or flow and can be estimated invasively as fractional flow reserve (14) or measured noninvasively with PET and ${ }^{15} \mathrm{O}$-labeled water $\left(\mathrm{H}_{2}{ }^{15} \mathrm{O}\right)(20)$ or ${ }^{13} \mathrm{~N}$-labeled ammonia $\left({ }^{13} \mathrm{NH}_{3}\right)$ (Fig. 3) (21).

However, because CFR is a ratio, factors that influence either the numerator or the denominator may affect its calculation (Table 1). Therefore, a low CFR value does not necessarily reflect a reduction of hyperemic flow, but it can be due to an abnormally elevated resting flow in the face of a normal hyperemic flow. This problem can be overcome, at least in part, by normalizing resting flow for the external cardiac workload, which is generally measured using the rate-pressure product (RPP) (22).

\section{MBF MEASUREMENT: RECENT ADVANCES}

\section{CMR}

Assessment of myocardial perfusion by means of CMR exploits the first-pass kinetics of T1-enhancing extracellular gadolinium chelates. During the first pass, the contrast medium diffuses in the interstitial space from the microvasculature, resulting in increased signal intensity proportional to the perfusion and blood volume of the tissue, size of extravascular compartment, and capillary permeability 


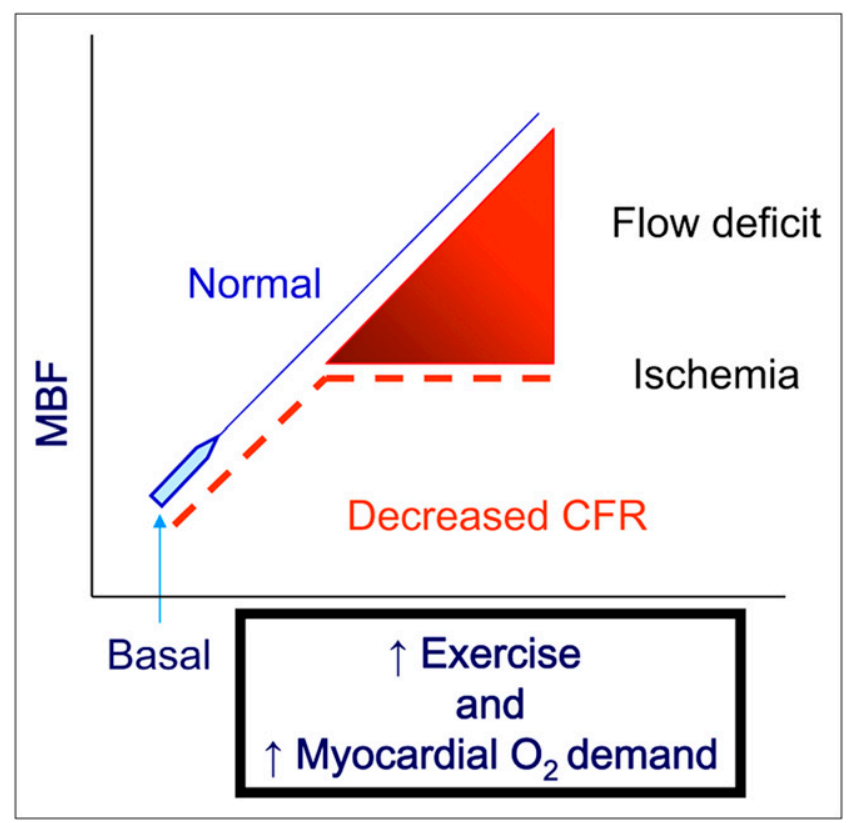

FIGURE 3. MBF can increase 3- to 4-fold during submaximal increase in cardiac workload to match increased myocardial oxygen demand. In patients with CAD, coronary stenoses reduce ability of coronary circulation to increase MBF to match increased workload, leading to myocardial ischemia. CFR is ratio of near-maximum MBF (measured during pharmacologic vasodilatation with intravenous adenosine or dipyridamole) to basal MBF. CFR can be measured noninvasively using PET to assess functional significance of coronary stenoses. In absence of coronary stenoses, reduced CFR reflects dysfunction of coronary microcirculation.

(23). Gadolinium chelates do not enter intact cell membranes and the volume of distribution is restricted to approximately $20 \%$ in normal viable myocardium (24). Different extravascular, extracellular contrast medium dose administration protocols and pulse sequences have been tested and validated in animal experiments (25-27), which have been subsequently translated into human studies (28-33).

The main issue in the design of a CMR perfusion pulse sequence is the trade-off between spatial and temporal resolution, linearity between signal intensity and contrast agent concentration, acquisition time, and signal-to-noise ratio. Different solutions to the above problems have been proposed over time by several groups to optimize performance (34). The standardization of protocols, pulse sequences, and models for semiquantitative or quantitative analysis is a necessary step to foster the application in everyday clinical cardiology or radiology practice.

The occurrence of image artifacts represents a limiting factor in clinical performance. The time-signal intensity curves may be degraded, and this prevents an accurate analysis. Potentially misleading artifacts are recognized in the subendocardial dark rims that can be visually misdiagnosed as perfusion defects.

Clinically, the widely used qualitative analysis consists of a visual examination of the homogeneous increase in signal during the first pass, followed by contrast washout; the detection of a delayed signal increase and persistently hypointense regions indicates that the blood supply is reduced by a high-grade stenosis $(35,36)$. Interpretation of the data can be semiquantitative, using the signal intensity index or the upslope rate $(32,37)$, or fully quantitative $(33,38)$. Fully quantitative perfusion analysis so far has not yet gained broad clinical use and is performed in highly specialized centers either by calculating various parameters of the tissue enhancement curves and correcting them by subtracting or scaling the arterial input function derived from the left ventricle $(27,32)$ or by using a modelindependent deconvolution $(39,40)$.

Two main approaches are currently under consideration for widespread clinical application: a stress hyperemic-only protocol and a stress-rest protocol for the assessment of CFR. The stress-rest protocol offers the advantage of improving the discrimination of dark rim artifacts from truly impaired perfusion in regions without prior myocardial infarction. However, the issue of residual ischemia in an infarcted region remains still incompletely addressed (35).

There is accruing evidence of the diagnostic performance of CMR stress perfusion with adenosine in patients with overt CAD (stenosis $>50 \%$ ) or in patients with a lower likelihood of disease or risk factors. Single-center $(32,35,37,41)$ and multicenter (42) studies have shown

\section{TABLE 1. Factors Affecting MBF}

Factors affecting measurement of resting MBF
Oxygen consumption (i.e., heart
rate, systemic blood pressure,
myocardial contractility)
Age
Sex
Endothelial dysfunction
Drugs
Presence of scar/fibrosis
(particularly with ${ }^{13} \mathrm{NH}_{3}$ )
Anemia

Factors affecting measurement of hyperemic MBF

Submaximal coronary vasodilatation

Caffeine and caffeine derivatives

Anatomic remodeling of the microcirculation

Increased microvascular tone

Increased extravascular compression/resistance

Coronary risk factors (diabetes, hyperlipidemia, smoke, hypertension)

Autonomic nervous system dysfunction

Systemic inflammation 
good sensitivity (range, 90\%-84\%) and specificity (range, $89 \%-58 \%$ ) for the detection of coronary CAD on a perpatient basis, compared with conventional coronary angiography. In a single-center study, in a cohort of 513 patients, the visual detection of ischemia at the CMR perfusion stress test was proven to be an independent predictor of hard cardiac events, whereas a normal stress test was associated with a low event rate (43). These findings will have to be verified in a multicenter, randomized trial and possibly with at least semiquantitative evaluation of the inducible perfusion abnormalities.

In the multiethnic study of atherosclerosis, a prospective study designed to evaluate risk factors and progression of cardiovascular disease, myocardial perfusion was assessed in 222 subjects (38). In 28 subjects, the reproducibility and repeatability of myocardial perfusion over an average of $1 \mathrm{y}$ was comparable with PET, with relative repeatability coefficients of $30 \%$ of mean at rest and $41 \%$ during hyperemia in the whole heart $(19,44)$.

The diagnostic performance of CMR perfusion assessed qualitatively has been compared head to head to more established noninvasive techniques such as SPECT in 18 centers in Europe and the United States for the detection of $\mathrm{CAD}$ using conventional coronary angiography as the standard of reference. CMR perfusion proved equivalent to gated SPECT, with $85 \%$ sensitivity and $67 \%$ specificity; the receiver-operating-characteristic (ROC) curve analysis favored CMR perfusion when compared with cumulative gated and nongated SPECT (42). In 2 studies, perfusion and CFR by CMR were tested against PET, the current noninvasive gold standard for the absolute measurement of perfusion; in both cases $(32,45)$, there was good agreement between the 2 techniques.

Qualitative and semiquantitative assessment of perfusion with CMR at $1.5 \mathrm{~T}$ has shown a moderate accuracy for the detection of CAD $(32,35,43,46)$. Low differences in contrast enhancement between normal and underperfused myocardium represent a limitation in the interpretation of the images. Scanners with higher magnetic field strength (e.g., $3 \mathrm{~T}$ ) have increased signal-to-noise ratio and contrast enhancement, which can be used to improve spatial resolution and image quality. On the other hand, increased field inhomogeneities can lead to more artifacts in perfusion imaging. In a pilot study in 65 patients, Cheng et al. reported that 3-T CMR perfusion imaging provides an improved diagnostic accuracy, sensitivity, and specificity for the detection of significant coronary stenosis perfusion imaging (30). Pulse sequences to perform CMR perfusion at $3 \mathrm{~T}$ are commercially available, and it is conceivable that in the near future more data will be available to analyze the performance of higher-magnetic-field-strength systems on a large scale.

\section{Subendocardial and Subepicardial MBF Measurement with PET}

Reduced subendocardial CFR in response to exercise or pharmacologically induced vasodilatation is a hallmark of flow-limiting stenoses. In the absence of coronary disease, this phenomenon has been demonstrated in patients with LV hypertrophy secondary to aortic stenosis (12). We have recently validated the PET measurement of transmural MBF distribution during pharmacologic stress in the left ventricle of normal thickness in a porcine model of ischemic heart disease (47). The use of a fully quantitative perfusion assessment is of clinical relevance because it circumvents the necessity of establishing a normal reference region of interest, which is particularly demanding in patients with multivessel disease and in all those conditions in which there is an increased heterogeneity in myocardial perfusion due to the presence of fibrosis or scarring. Differences in the composition of the different tissues surrounding the subendocardium and the subepicardium (blood vs. chest wall and lung tissue) can account for the small systematic difference in the agreement with microspheres between subendocardial and subepicardial flow (Fig. 4). The kinetic model used to quantify MBF contains an intrinsic correction for spillover from arterial blood activity but not for that from adjacent tissue activity; as a result, there is a slight overestimation in subepicardial MBF relative to that in the subendocardium.

Short- and Long-Term Reproducibility of Perfusion Measurement with PET. Different studies have tested the short-term reproducibility of MBF measurements using PET with ${ }^{13} \mathrm{NH}_{3}$ and $\mathrm{H}_{2}{ }^{15} \mathrm{O}(48,49)$. Repeated measurements of resting and hyperemic MBF using intravenous dipyridamole and adenosine during the same study session were not significantly different, demonstrating the validity of the technique. The variability of hyperemic flow was larger, as indicated by the larger repeatability coefficient, and was paralleled by a greater variability of the RPP. This

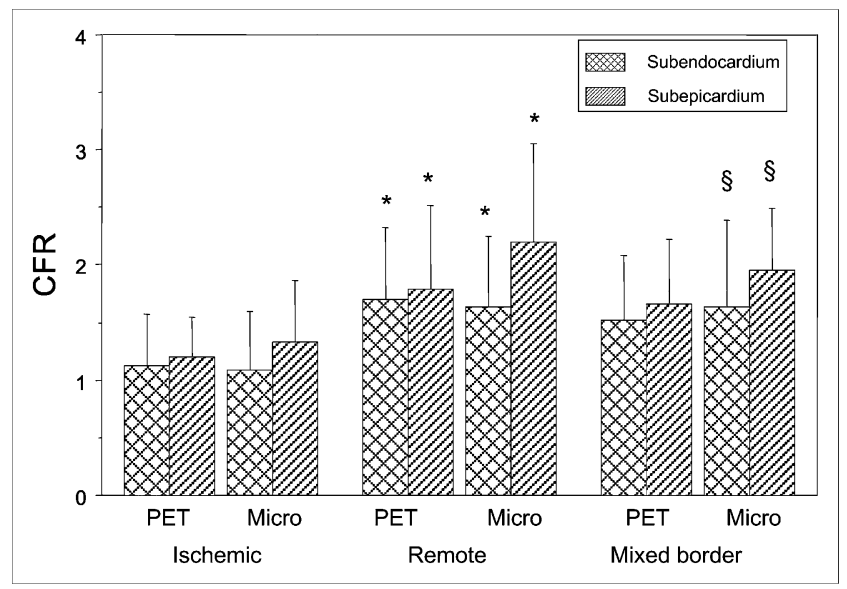

FIGURE 4. Comparison of CFR between subepicardium and subendocardium in 3 territories (ischemic, remote, mixed border) of left ventricle in pigs with regional myocardial ischemia. ${ }^{*}$ Remote vs. ischemic, $P<0.05$. §Mixed border vs. ischemic, $P<0.05$. Micro $=$ microspheres. (Reproduced with permission of (47).) 
could mean that the greater variability of MBF during stress is more likely due to a variable response to vasodilators rather than to a larger measurement error. In a subsequent study from the same group, the authors tested the feasibility and reproducibility of MBF measurement during supine bicycle exercise. The study results demonstrated the feasibility of this protocol, which was found at least as repeatable as using adenosine stress (50).

These results demonstrate that serial MBF measurements with PET can be used to quantify the effect of various interventions on $\mathrm{MBF}$ and flow reserve.

More recently, Jagathesan et al. (51) have tested the long-term reproducibility of MBF measurement at rest and after dobutamine stress in patients with stable CAD using PET with $\mathrm{H}_{2}{ }^{15} \mathrm{O}$. Dobutamine induced reproducible changes in both global and regional MBF and flow reserve over $24 \mathrm{wk}$. The reproducibility of MBF and flow reserve with dobutamine was comparable with the short-term repeatability reported for adenosine and physical exercise in healthy subjects. In a subsequent study, the same authors (52) compared the relationship between dobutamine MBF and stenosis severity in patients with single-vessel CAD. In patients with stenoses less than or equal to $69 \%$, MBF during dobutamine stress in territories subtended by diseased arteries was comparable to flow in remote myocardium subtended by nondiseased arteries. On the other hand, MBF during dobutamine stress in territories subtended by coronary arteries with stenoses greater than or equal to $70 \%$ was significantly lower than flow in remote myocardium subtended by nondiseased arteries. An inverse relationship between dobutamine MBF and stenosis severity was found both in ischemic and in remote territories. The latter observation is consistent with previous reports demonstrating abnormal CFR with vasodilator stressors in myocardial segments subtended by angiographically normal coronary arteries and most likely reflects concomitant microvascular dysfunction.

\section{Differences Among Stressors}

Mechanistic differences between pharmacologic stressors may offer different clinical benefits. In a recent study, the effects of dobutamine and adenosine on absolute MBF and CFR were compared in a cohort of patients with CAD and a group of healthy volunteers (19). In healthy volunteers, $\mathrm{MBF}$ and flow reserve during adenosine were higher than during dobutamine. In patients, adenosine produced a more significant flow difference between ischemic and remote myocardial territories than did dobutamine, which achieved flow heterogeneity to a lesser extent and only in patients with severe coronary artery stenosis $(75 \%)$. However, in myocardial segments subtended by a significant coronary stenosis, dobutamine achieved greater hyperemia than adenosine stress and CFR values similar to adenosine stress (Fig. 5). This greater hyperemia with dobutamine may indicate greater collateral recruitment or reflect the greater propensity for vasodilator agents to induce coronary steal.

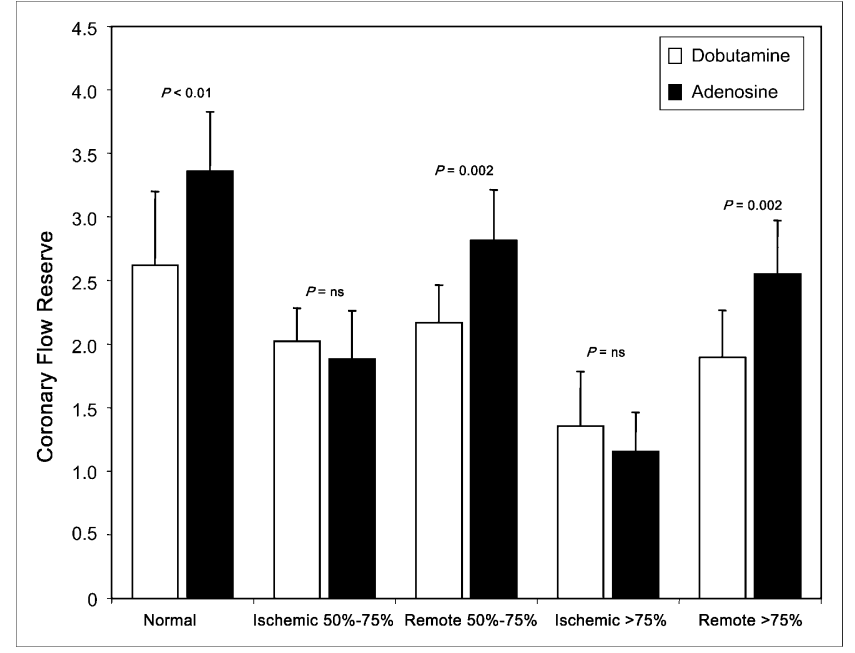

FIGURE 5. CFR measured by PET with $\mathrm{H}_{2}{ }^{15} \mathrm{O}$ during dobutamine and adenosine stress in healthy volunteers and patients with CAD. Data demonstrate that similar flow responses are achieved with 2 stressors in ischemic territories, whereas flow responses in remote and normal myocardium are different. ns = not significant. (Reproduced with permission of (19).)

Therefore, the higher diagnostic sensitivity quoted with vasodilator stress primarily reflects the greater hyperemia seen with these agents in remote myocardial segments. In summary, this study using quantitative perfusion imaging demonstrated that adenosine stress has higher sensitivity and dobutamine has higher specificity.

\section{MBF AND OVERT CAD}

The methodologic aspects and main clinical applications of MBF measurement have been reviewed recently (7).

Endothelial dysfunction is an early marker of coronary atherosclerosis and a predictor of cardiovascular events $(53,54)$. The techniques generally used to test endothelial function are invasive and involve intracoronary administration of drugs during coronary angiography. More recently, measurement of MBF with PET in response to a cold pressure test (CPT) has been used as a noninvasive tool for the assessment of endothelial function (55). The accuracy, reproducibility, and clinical value of this approach have been validated in several studies by different groups (56-59).

This technique has been used to demonstrate endothelial dysfunction in patients with insulin resistance (60) and its improvement with euglycemic control and insulin treatment in patients with type 2 diabetes (61); this technique also shows the effect of thiazolidinedione therapy in insulin-resistant individuals (62). Schindler et al. demonstrated that the CPT-induced increase in MBF ( $\delta$-MBF) was significantly less in diabetic patients than in controls (mean \pm $\mathrm{SD}, 0.07 \pm 0.07$ vs. $0.25 \pm 0.12 \mathrm{~mL} / \mathrm{min} / \mathrm{g} ; P<0.001)$ (61). After treatment with glyburide and metformin, which 
significantly decreased plasma glucose concentrations in diabetic patients, there was a significant increase in $\delta-\mathrm{MBF}$ in response to CPT $(0.20 \pm 0.16$ from $0.07 \pm 0.07 \mathrm{~mL} /$ $\mathrm{min} / \mathrm{g} ; P<0.001$ ). Quinones et al. (62) measured MBF at rest and after CPT in insulin-resistant patients and controls at baseline and after 3 mo of thiazolidinedione therapy. At baseline, MBF response to CPT increased by $47.6 \%$ from resting values in insulin-sensitive patients and by $14.4 \%$ in insulin-resistant patients. During thiazolidinedione therapy, insulin sensitivity improved, fasting plasma insulin levels decreased, and MBF responses to CPT normalized.

Morita et al. (63) studied 15 young smokers with no evidence of heart disease or other cardiovascular risk factors and a group of age-matched nonsmokers $(n=12)$ to assess the effect of smoking cessation on coronary endothelial function. At baseline, MBF during the CPT in smokers was lower than that in nonsmokers $(0.90 \pm 0.19$ vs. $1.12 \pm 0.28 \mathrm{~mL} / \mathrm{g} / \mathrm{min} ; P<0.05)$. However, 1 mo after smoking cessation, compared with baseline, MBF during the CPT increased $(0.90 \pm 0.19$ vs. $1.02 \pm 0.22 \mathrm{~mL} / \mathrm{g} / \mathrm{min}$; $P<0.01$ ), and the improvement was preserved at $6 \mathrm{mo}$ after smoking cessation.

Finally, similarly to the data obtained with the invasive approach (54), impaired MBF response to CPT assessed by PET is associated with cardiovascular events at follow-up (55).

PET has been used to demonstrate the effect of cardiac resynchronization therapy on the redistribution of MBF in patients with nonischemic and ischemic cardiomyopathy (64). Wielepp et al. (65) studied $26 \mathrm{CAD}$ patients with untreated hypercholesterolemia. MBF was measured at rest and at $4.6 \pm 1.8$ mo after statin therapy. Minimum coronary vascular resistance (MCR) and CFR were determined. Segments were divided into those with normal or nearnormal MBF during adenosine (i.e., $\geq 2.0 \mathrm{~mL} / \mathrm{min} / \mathrm{g}$ ) and those with $\mathrm{MBF}$ during adenosine (i.e., $<2.0 \mathrm{~mL} / \mathrm{min} / \mathrm{g}$ ). After treatment with statin, no significant changes in $\mathrm{MBF}$, flow reserve, and MCR were found in normal segments. However, initially abnormal segments showed significant improvements in MCR $(15 \% ; P<0.0001)$ and $\mathrm{MBF}$ during adenosine $(30 \% ; P<0.0001)$ after therapy.

The relationship between resting and hyperemic MBF and severity of coronary stenoses in patients with coronary disease has been studied using $\mathrm{H}_{2}{ }^{15} \mathrm{O}$ and ${ }^{13} \mathrm{NH}_{3}$. The widespread clinical use of this approach for the evaluation of the functional significance of coronary stenoses has been limited by the fact that both $\mathrm{H}_{2}{ }^{15} \mathrm{O}$ and ${ }^{13} \mathrm{NH}_{3}$ require a cyclotron on site for their production. The generatorproduced cationic potassium analog ${ }^{82} \mathrm{Rb}$ represents an attractive alternative because it does not require a cyclotron on-site and has a short half-life (78 s). Early experimental studies have shown that, although ${ }^{82} \mathrm{Rb}$ with PET allows detection of myocardial ischemia, quantification of $\mathrm{MBF}$ using this tracer is limited by the heavy dependence of its myocardial extraction at higher flow rates (66). Because the myocardial extraction of ${ }^{82} \mathrm{Rb}$ decreases significantly with increasing flow rates, the calculated hyperemic flow with vasodilator stress may underestimate the actual flow rate. Therefore, quantification of regional $\mathrm{MBF}$ with ${ }^{82} \mathrm{Rb}$ may be inaccurate, particularly during hyperemia or in metabolically impaired myocardium. In addition, the high positron energy $(3.15 \mathrm{MeV})$ of this radionuclide results in relatively poor image quality and in a reduced spatial resolution due to its relatively long positron track. More recently, however, a novel approach, based on a generalized factor analysis of dynamic sequences (GFADS) and a 2-compartment kinetic model, has been developed for the quantification of regional $\mathrm{MBF}$ with ${ }^{82} \mathrm{Rb}$. Using this approach, Anagnostopoulos et al. (67) have studied the relationship between stenosis severity, assessed with quantitative coronary angiography, and $\mathrm{MBF}$, measured with ${ }^{82} \mathrm{Rb}$ in patients with $\mathrm{CAD}$. The results of this investigation were consistent with those previously reported using $\mathrm{H}_{2}{ }^{15} \mathrm{O}$ and ${ }^{13} \mathrm{NH}_{3}(21,22)$.

In another recent PET study, Lortie et al. (68) have compared MBF measured with ${ }^{13} \mathrm{NH}_{3}$ and ${ }^{82} \mathrm{Rb}$ in the same groups of healthy volunteers and patients with CAD. The $\mathrm{MBF}$ values obtained with ${ }^{82} \mathrm{Rb}$ using a 1-compartment model were in good agreement with those obtained with ${ }^{13} \mathrm{NH}_{3}$ both in healthy volunteers and in patients with CAD. Altogether, these preliminary studies in relatively small groups of subjects provide evidence that PET with ${ }^{82} \mathrm{Rb}$ allows the quantification of regional MBF. This approach may represent a valid alternative to SPECT myocardial perfusion imaging and in addition may allow detection of balanced or global MBF reductions.

The prognostic value of myocardial perfusion imaging using ${ }^{82} \mathrm{Rb}$ and PET has been evaluated by Yoshinaga et al. (69) in 367 patients who were followed for $3.1 \pm 0.9 \mathrm{y}$. Rest and stress myocardial perfusion were assessed semiquantitatively, and patients were stratified according to their summed stress scores. The severity of the summed stress scores was shown to have significant prognostic value in predicting major adverse cardiac events, including death and myocardial infarction, and total cardiac events, including revascularization and hospitalization.

In a cohort of 695 patients with an intermediate likelihood of CAD, Schenker et al. (70) have assessed the added value of combining coronary artery calcium (CAC) scoring with the semiquantitative analysis of rest-stress ${ }^{82} \mathrm{Rb}$ perfusion using a hybrid PET/CT scanner. The results of this study showed that although increasing CAC content is generally predictive of a higher likelihood of ischemia, its absence does not completely eliminate the possibility of flow-limiting CAD. Importantly, a stepwise increase occurs in the risk of adverse events with increasing CAC scores in patients with and without ischemia on PET myocardial perfusion imaging. Finally, in 100 consecutive patients, Siegrist et al. (71) have evaluated the impact of myocardial perfusion imaging with ${ }^{13} \mathrm{NH}_{3}$ and PET on clinical decision making and cost effectiveness in a population with high prevalence of CAD. The results of this study have shown 
that PET with ${ }^{13} \mathrm{NH}_{3}$ is cost-effective and affects patient management.

\section{MBF AND CORONARY MICROVASCULAR DYSFUNCTION}

There is no technique that enables direct visualization of coronary microcirculation in vivo in humans. Therefore, its assessment relies on the measurement of parameters that reflect its functional status, such as MBF and CFR. CFR is an integrated measure of flow through both the large epicardial coronary arteries and the microcirculation (7).

In the absence of obstructive stenoses on the epicardial arteries, a reduced CFR is a marker of coronary microvascular dysfunction. Although a single cutoff value of CFR (e.g., $\leq 2.0$ ) below which microvascular function is deemed abnormal would be useful clinically, in healthy humans, CFR varies according to age and sex (20). Therefore, it is essential to compare CFR data in patients with those obtained in age- and sex-matched healthy subjects. In addition, resting MBF is linearly related to cardiac work. Therefore, when comparing different patients it is important to correct resting MBF for the main determinants of external cardiac workload (i.e., blood pressure and heart rate $[R P P])$. A corrected CFR can then be calculated by dividing hyperemic flow by RPP-corrected resting flow (22). More complex is the assessment of coronary microvascular dysfunction in territories subtended by stenotic coronary arteries in which the evaluation of microvascular function depends on the clinical context.

As proposed by Camici and Crea (5), coronary microvascular dysfunction can be classified in the following 4 groups: coronary microvascular dysfunction occurring in the absence of obstructive epicardial CAD and myocardial diseases (type A), coronary microvascular dysfunction occurring in the context of cardiomyopathies (type B), coronary microvascular dysfunction occurring in the presence of obstructive epicardial CAD (type C), and iatrogenic coronary microvascular dysfunction (type D).

Lately, several studies have expanded our understanding of coronary microvascular dysfunction in different clinical settings. In moderately hypertensive patients with stable $\mathrm{CAD}$, the effect of treatment with the angiotensin receptor blocker valsartan on MBF was measured with PET and ${ }^{13} \mathrm{NH}_{3}$ (72). Significant improvements in MBF both after CPT and in response to pharmacologic vasodilatation with adenosine could be demonstrated after 1 and 16 wk of treatment. The improvement in MBF preceded the reduction of blood pressure, suggesting direct beneficial effects on microvascular function. In another study in hypertensive patients with LV hypertrophy, the effect of treatment with the angiotensin-converting enzyme inhibitor perindopril, in combination with the diuretic indapamide on resting and hyperemic (adenosine) MBF, was assessed. Compared with baseline, after 6 mo of treatment both systolic and diastolic blood pressures were significantly reduced and there was an increase in MBF and a significant reduction of LV mass (73).
In a randomized, double-blind, placebo-controlled study in 26 nondiabetic patients with familial combined hyperlipidemia, Naoumova et al. (74) tested the effect of treatment with the insulin sensitizer thiazolidinedione pioglitazone added to conventional lipid-lowering therapy on myocardial glucose use and blood flow using PET with ${ }^{18} \mathrm{~F}-\mathrm{FDG}$ and $\mathrm{H}_{2}{ }^{15} \mathrm{O}$, respectively. After 16 wk of treatment, there was evidence of significant increases in myocardial glucose use and hyperemic blood flow with adenosine, again suggesting improved coronary microvascular function.

In a double-blind, placebo-controlled pilot trial, 16 consecutive patients with idiopathic-dilated cardiomyopathy were randomized to treatment with either carvedilol or placebo (75). Regional MBF was measured at rest and after intravenous injection of dipyridamole using PET with ${ }^{13} \mathrm{NH}_{3}$ at baseline and after 6 mo of treatment. Absolute MBF did not change significantly after carvedilol or placebo treatment, although hyperemic blood flow showed a trend toward increase in patients receiving active treatment. CFR significantly increased after carvedilol treatment; it remained unchanged after placebo, and stress-induced regional perfusion defects decreased after active treatment (75).

Previous PET studies have demonstrated that maximum MBF and flow reserve are severely blunted in patients with hypertrophic cardiomyopathy (HCM), and the severity of the impairment predicts ventricular remodeling and prognosis (Fig. 6) (76-78). In a recent paper by Petersen et al. (31), the correlation between degree of hypertrophy, MBF at rest and during hyperemia (adenosine), and myocardial fibrosis was assessed using MRI in 35 patients with HCM. This study provided novel evidence that hyperemic MBF decreased with increasing end-diastolic wall thickness and preferentially in the subendocardial layers. The frequency with which subendocardial blood flow fell below subepicardial blood flow was directly proportional to wall thickness and the incidence of fibrosis assessed by gadolinium late enhancement (31). In another study of 18 patients with HCM, PET with $\mathrm{H}_{2}{ }^{15} \mathrm{O}$ was used to measure subepicardial and subendocardial blood flow and MRI was used to determine LV mass and volumes; LV outflow tract gradient was measured at cardiac catheterization. The subendocardialto-subepicardial flow ratio decreased significantly during hyperemia, and the pattern was similar for the hypertrophied septum and lateral wall. Hyperemic flow was inversely correlated with LV outflow tract gradient, terminal pro-brain natriuretic peptide, left atrial volume index, and ventricular mass. These findings suggest that, in addition to microvascular remodeling and vessel rarefaction, extravascular compressive forces contribute to microvascular dysfunction in patients with HCM (79).

In a preliminary study in healthy volunteers, Kaufmann et al. (80) demonstrated that the maximum MBF response to intravenous adenosine measured with PET and $\mathrm{H}_{2}{ }^{15} \mathrm{O}$ in humans was constrained by what could be a neurally 
A
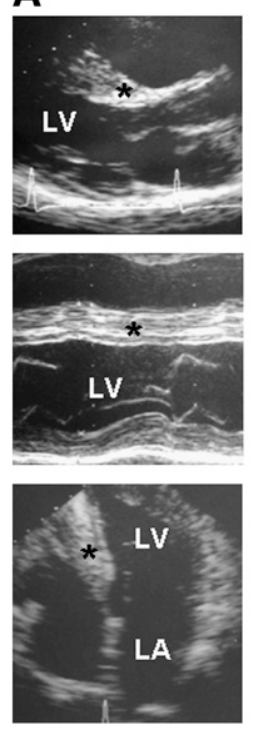

B
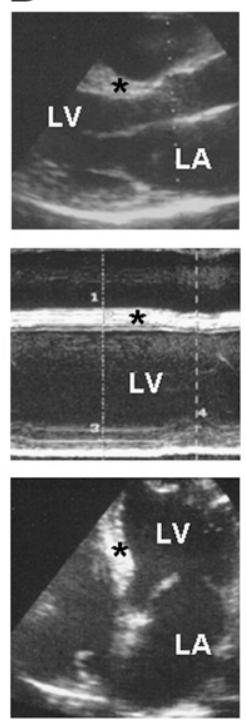

C

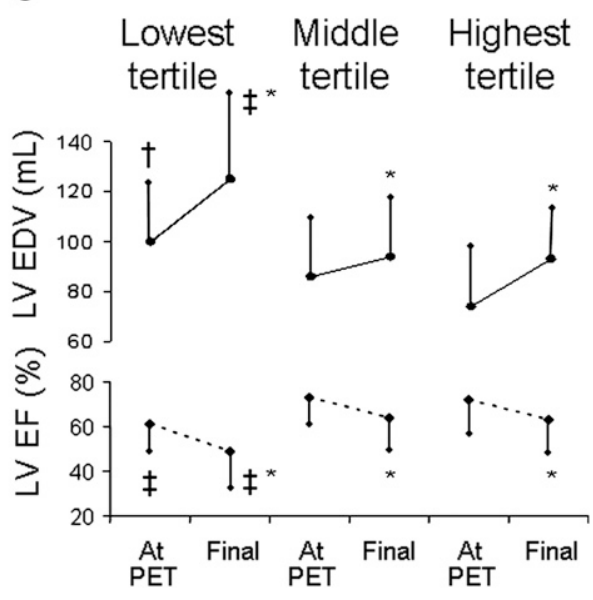

FIGURE 6. Severe impairment of CFR predicts LV remodeling at followup in patients with HCM. Stop frames of echocardiograms obtained at time of PET scan (at age $33 \mathrm{y}$ ) (A) and at final evaluation (at age 42 y) (B) in HCM patient with missense mutations of myosin $\beta$-heavy chain and of myosin binding protein C genes (Arg723Cys and Glu165Asp, respectively). Patient had severely impaired flow response to dipyridamole (MBF was $0.71 \mathrm{~mL} / \mathrm{min} / \mathrm{g}$ under resting conditions and only 1.06 $\mathrm{mL} / \mathrm{min} / \mathrm{g}$ following intravenous dipyridamole). Comparison of two echocardiograms at baseline $(A)$ and at end of follow-up (B) shows progression of LV cavity enlargement and systolic impairment, with regression of septal hypertrophy. This patient had no functional limitation at time of PET; during $9 \mathrm{y}$ of follow-up, congestive symptoms progressively developed and at final evaluation patient was severely limited, with dyspnea on minimal effort. Each set of echocardiographic images (top to bottom) consists of parasternal long-axis and apical 4-chamber views. ${ }^{*}$ Interventricular septum. $L A=$ left atrium; $L V=$ left ventricle. (C) Comparison of LV end-diastolic volume and ejection fraction at time of measurement of MBF and flow reserve (dipyridamole) using PET and at final evaluation according to tertiles of dipyridamole MBF. Vertical bars indicate mean \pm SD for each group. ${ }^{*} P<0.05$ vs. same group at time of PET scan. ${ }^{\dagger} P<0.05$ vs. patients in highest tertile. ${ }^{\ddagger} P<0.05$ vs. patients in other tertiles. EDV = end-diastolic volume; EF = ejection fraction. (Reproduced with permission of (77).)

mediated vasoconstriction of resistance vessels. This latter was paradoxically relieved by systemic infusion of the nitric oxide synthase inhibitor L-NMMA, as suggested by the lack of flow increase after L-NMMA observed in transplant recipients whose hearts were denervated for several months after surgery. In a subsequent study using the same methodology, the same authors have shown that the systemic infusion of L-NMMA in patients with CAD significantly increased the MBF response to adenosine in territories subtended by stenotic ( $\geq 70 \%$ luminal diameter) coronary arteries $(81)$. The notion that the flow response to adenosine or dipyridamole does not represent the maximum flow achievable in the coronary system has been previously demonstrated in animals $(82,83)$. Furthermore, in a study in healthy human volunteers, in whom MBF was measured noninvasively by means of PET with $\mathrm{H}_{2}{ }^{15} \mathrm{O}$, Lorenzoni et al. (84) showed that the flow response to dipyridamole was increased by approximately $40 \%$ when the study was repeated during pharmacologic blockade of peripheral $\alpha_{1}$ adrenoceptors. Further studies are needed to ascertain whether the findings of this investigation offer the possibility of devising some new form of treatment to improve myocardial perfusion in patients with chronic CAD.

\section{CONCLUSION}

PET provides robust and reproducible measurements of regional MBF in milliliters per minute per gram of tissue. Experiments in animals and humans have demonstrated that CMR, compared with PET, also has the ability to provide accurate quantification of regional MBF, although its use in clinical studies is still limited. Compelling evidence suggests that in many instances abnormalities of global myocardial perfusion are demonstrated in individuals without $\mathrm{CAD}$ with either coronary risk factors or with different myocardial diseases. In this context, measurement of MBF gives unique diagnostic information regarding the function of the coronary microcirculation and provides a quantitative surrogate endpoint against which the efficacy of treatments can be established.

\section{REFERENCES}

1. Rosamond W, Flegal K, Furie K, et al. Heart disease and stroke statistics: 2008 update-a report from the American Heart Association Statistics Committee and Stroke Statistics Subcommittee. Circulation. 2008;117:e25-e146.

2. Hunt SA, Abraham WT, Chin MH, et al. ACC/AHA 2005 guideline update for the diagnosis and management of chronic heart failure in the adult: a report of the American College of Cardiology/American Heart Association Task Force on Practice Guidelines (writing committee to update the 2001 guidelines for the evaluation and management of heart failure)-developed in collaboration with the American College of Chest Physicians and the International Society for Heart and Lung Transplantation: endorsed by the Heart Rhythm Society. Circulation. 2005;112:e154-e235.

3. Uren NG, Marraccini P, Gistri R, de Silva R, Camici PG. Altered coronary vasodilator reserve and metabolism in myocardium subtended by normal arteries in patients with coronary artery disease. J Am Coll Cardiol. 1993;22:650-658.

4. Klocke FJ, Baird MG, Lorell BH, et al. ACC/AHA/ASNC guidelines for the clinical use of cardiac radionuclide imaging: executive summary-a report of the American College of Cardiology/American Heart Association Task Force on Practice Guidelines (ACC/AHA/ASNC Committee to Revise the 1995 Guidelines for the Clinical Use of Cardiac Radionuclide Imaging). Circulation. 2003;108:1404-1418.

5. Camici PG, Crea F. Coronary microvascular dysfunction. N Engl J Med. 2007; 356:830-840. 
6. Dorbala S, Hassan A, Heinonen T, Schelbert HR, Di Carli MF. Coronary vasodilator reserve and Framingham risk scores in subjects at risk for coronary artery disease. J Nucl Cardiol. 2006;13:761-767.

7. Kaufmann PA, Camici PG. Myocardial blood flow measurement by PET: technical aspects and clinical applications. J Nucl Med. 2005;46:75-88.

8. Araujo LI, Lammertsma AA, Rhodes CG, et al. Noninvasive quantification of regional myocardial blood flow in coronary artery disease with oxygen-15labeled carbon dioxide inhalation and positron emission tomography. Circulation. 1991;83:875-885.

9. Chilian WM. Coronary microcirculation in health and disease: summary of an NHLBI workshop. Circulation. 1997;95:522-528.

10. Klassen GA, Armour JA, Garner JB. Coronary circulatory pressure gradients. Can J Physiol Pharmacol. 1987;65:520-531.

11. Duncker DJ, Bache RJ. Regulation of coronary blood flow during exercise. Physiol Rev. 2008;88:1009-1086.

12. Rajappan K, Rimoldi OE, Dutka DP, et al. Mechanisms of coronary microcirculatory dysfunction in patients with aortic stenosis and angiographically normal coronary arteries. Circulation. 2002;105:470-476.

13. Westerhof N, Boer C, Lamberts RR, Sipkema P. Cross-talk between cardiac muscle and coronary vasculature. Physiol Rev. 2006;86:1263-1308.

14. De Bruyne B, Pijls NH, Barbato E, et al. Intracoronary and intravenous adenosine 5'-triphosphate, adenosine, papaverine, and contrast medium to assess fractional flow reserve in humans. Circulation. 2003;107:1877-1883.

15. Vanhoutte PM. Endothelial control of vasomotor function: from health to coronary disease. Circ J. 2003;67:572-575.

16. DeFily DV, Chilian WM. Coronary microcirculation: autoregulation and metabolic control. Basic Res Cardiol. 1995;90:112-118.

17. Belardinelli L, Linden J, Berne RM. The cardiac effects of adenosine. Prog Cardiovasc Dis. 1989;32:73-97.

18. Stanley WC. Myocardial energy metabolism during ischemia and the mechanisms of metabolic therapies. J Cardiovasc Pharmacol Ther. 2004;9(suppl 1): S31-S45.

19. Jagathesan R, Barnes E, Rosen SD, Foale RA, Camici PG. Comparison of myocardial blood flow and coronary flow reserve during dobutamine and adenosine stress: implications for pharmacologic stress testing in coronary artery disease. J Nucl Cardiol. 2006;13:324-332.

20. Chareonthaitawee P, Kaufmann PA, Rimoldi O, Camici PG. Heterogeneity of resting and hyperemic myocardial blood flow in healthy humans. Cardiovasc Res. 2001;50:151-161.

21. Di Carli M, Czernin J, Hoh CK, et al. Relation among stenosis severity, myocardial blood flow, and flow reserve in patients with coronary artery disease. Circulation. 1995;91:1944-1951.

22. Uren NG, Melin JA, De Bruyne B, Wijns W, Baudhuin T, Camici PG. Relation between myocardial blood flow and the severity of coronary-artery stenosis. N Engl J Med. 1994;330:1782-1788.

23. Tong CY, Prato FS, Wisenberg G, et al. Measurement of the extraction efficiency and distribution volume for Gd-DTPA in normal and diseased canine myocardium. Magn Reson Med. 1993;30:337-346.

24. Schwitter J, Saeed M, Wendland MF, et al. Influence of severity of myocardial injury on distribution of macromolecules: extravascular versus intravascular gadolinium-based magnetic resonance contrast agents. J Am Coll Cardiol. 1997;30:1086-1094.

25. Christian T, Aletras AH, Arai AE. Estimation of absolute myocardial blood flow during first-pass MR perfusion imaging using a dual-bolus injection technique: comparison to single-bolus injection method. J Magn Reson Imaging. 2008; 27:1271-1277.

26. Goldstein TA, Jerosch-Herold M, Misselwitz B, Zhang H, Gropler RJ, Zheng J. Fast mapping of myocardial blood flow with MR first-pass perfusion imaging. Magn Reson Med. 2008;59:1394-1400.

27. Lee DC, Simonetti OP, Harris KR, et al. Magnetic resonance versus radionuclide pharmacological stress perfusion imaging for flow-limiting stenoses of varying severity. Circulation. 2004;110:58-65.

28. Hsu LY, Rhoads KL, Holly JE, Kellman P, Aletras AH, Arai AE. Quantitative myocardial perfusion analysis with a dual-bolus contrast-enhanced first-pass MRI technique in humans. J Magn Reson Imaging. 2006;23:315-322.

29. Köstler H, Ritter C, Lipp M, Beer M, Hahn D, Sandstede J. Comparison of different contrast agents and doses for quantitative MR myocardial perfusion imaging. J Magn Reson Imaging. 2008;28:382-389.

30. Cheng AS, Pegg TJ, Karamitsos TD, et al. Cardiovascular magnetic resonance perfusion imaging at 3-tesla for the detection of coronary artery disease: a comparison with 1.5-tesla. J Am Coll Cardiol. 2007;49:2440-2449.

31. Petersen SE, Jerosch-Herold M, Hudsmith LE, et al. Evidence for microvascular dysfunction in hypertrophic cardiomyopathy: new insights from multiparametric magnetic resonance imaging. Circulation. 2007;115:2418-2425.
32. Schwitter J, Nanz D, Kneifel S, et al. Assessment of myocardial perfusion in coronary artery disease by magnetic resonance: a comparison with positron emission tomography and coronary angiography. Circulation. 2001;103:22302235 .

33. Rosen BD, Lima JA, Nasir K, et al. Lower myocardial perfusion reserve is associated with decreased regional left ventricular function in asymptomatic participants of the multi-ethnic study of atherosclerosis. Circulation. 2006; 114:289-297.

34. Kellman P, Arai AE. Imaging sequences for first pass perfusion: a review. J Cardiovasc Magn Reson. 2007;9:525-537.

35. Klem I, Heitner JF, Shah DJ, et al. Improved detection of coronary artery disease by stress perfusion cardiovascular magnetic resonance with the use of delayed enhancement infarction imaging. J Am Coll Cardiol. 2006;47:1630-1638.

36. Ingkanisorn WP, Kwong RY, Bohme NS, et al. Prognosis of negative adenosine stress magnetic resonance in patients presenting to an emergency department with chest pain. J Am Coll Cardiol. 2006;47:1427-1432.

37. Al-Saadi N, Nagel E, Gross M, et al. Noninvasive detection of myocardial ischemia from perfusion reserve based on cardiovascular magnetic resonance. Circulation. 2000;101:1379-1383.

38. Wang L, Jerosch-Herold M, Jacobs DR Jr, Shahar E, Folsom AR. Coronary risk factors and myocardial perfusion in asymptomatic adults: the multi-ethnic study of atherosclerosis (MESA). J Am Coll Cardiol. 2006;47:565-572.

39. Jerosch-Herold M, Swingen C, Seethamraju RT. Myocardial blood flow quantification with MRI by model-independent deconvolution. Med Phys. 2002;29:886-897.

40. Hsu LY, Kellman P, Arai AE. Nonlinear myocardial signal intensity correction improves quantification of contrast-enhanced first-pass MR perfusion in humans. J Magn Reson Imaging. 2008;27:793-801.

41. Cury RC, Cattani CAM, Gabure LAG, et al. Diagnostic performance of stress perfusion and delayed-enhancement MR imaging in patients with coronary artery disease. Radiology. 2006;240:39-45.

42. Schwitter J, Wacker CM, van Rossum AC, et al. MR-IMPACT: comparison of perfusion-cardiac magnetic resonance with single-photon emission computed tomography for the detection of coronary artery disease in a multicentre, multivendor, randomized trial. Eur Heart J. 2008;29:480-489.

43. Jahnke C, Nagel E, Gebker R, et al. Prognostic value of cardiac magnetic resonance stress tests: adenosine stress perfusion and dobutamine stress wall motion imaging. Circulation. 2007;115:1769-1776.

44. Jerosch-Herold M, Vazquez G, Wang L, Jacobs DR Jr, Folsom AR. Variability of myocardial blood flow measurements by magnetic resonance imaging in the multi-ethnic study of atherosclerosis. Invest Radiol. 2008;43:155-161.

45. Ibrahim T, Nekolla SG, Schreiber K, et al. Assessment of coronary flow reserve: comparison between contrast-enhanced magnetic resonance imaging and positron emission tomography. J Am Coll Cardiol. 2002;39:864-870.

46. Nagel E, Thouet T, Klein C, et al. Noninvasive determination of coronary blood flow velocity with cardiovascular magnetic resonance in patients after stent deployment. Circulation. 2003;107:1738-1743.

47. Rimoldi O, Schafers KP, Boellaard R, et al. Quantification of subendocardial and subepicardial blood flow using ${ }^{15} \mathrm{O}$-labeled water and PET: experimental validation. J Nucl Med. 2006;47:163-172.

48. Kaufmann PA, Gnecchi-Ruscone T, Yap JT, Rimoldi O, Camici PG. Assessment of the reproducibility of baseline and hyperemic myocardial blood flow measurements with ${ }^{15}$ O-labeled water and PET. J Nucl Med. 1999;40:18481856.

49. Nagamachi S, Czernin J, Kim AS. Reproducibility of measurement of regional resting and hyperemic myocardial blood flow assessed with PET. J Nucl Med. 1996;37:1626-1631.

50. Wyss CA, Koepfli P, Mikolajczyk K, Burger C, von Schulthess GK, Kaufmann PA. Bicycle exercise stress in PET for assessment of coronary flow reserve: repeatability and comparison with adenosine stress. J Nucl Med. 2003;44:146154.

51. Jagathesan R, Kaufmann PA, Rosen SD, et al. Assessment of the long-term reproducibility of baseline and dobutamine-induced myocardial blood flow in patients with stable coronary artery disease. J Nucl Med. 2005;46:212-219.

52. Jagathesan R, Barnes E, Rosen SD, Foale R, Camici PG. Dobutamine-induced hyperaemia inversely correlates with coronary artery stenosis severity and highlights dissociation between myocardial blood flow and oxygen consumption. Heart. 2006;92:1230-1237.

53. Zeiher AM, Drexler H, Wollschlager H, Just H. Endothelial dysfunction of the coronary microvasculature is associated with coronary blood flow regulation in patients with early atherosclerosis. Circulation. 1991;84:1984-1992.

54. Suwaidi JA, Hamasaki S, Higano ST, Nishimura RA, Holmes DR Jr, Lerman A. Long-term follow-up of patients with mild coronary artery disease and endothelial dysfunction. Circulation. 2000;101:948-954. 
55. Schindler TH, Nitzsche EU, Schelbert HR, et al. Positron emission tomographymeasured abnormal responses of myocardial blood flow to sympathetic stimulation are associated with the risk of developing cardiovascular events. J Am Coll Cardiol. 2005;45:1505-1512.

56. Siegrist PT, Gaemperli O, Koepfli P, et al. Repeatability of cold pressor test-induced flow increase assessed with $\mathrm{H}_{2}{ }^{15} \mathrm{O}$ and PET. J Nucl Med. 2006;47:1420-1426.

57. Schindler TH, Zhang XL, Vincenti G, et al. Diagnostic value of PET-measured heterogeneity in myocardial blood flows during cold pressor testing for the identification of coronary vasomotor dysfunction. J Nucl Cardiol. 2007;14:688-697.

58. Schindler TH, Zhang XL, Prior JO, et al. Assessment of intra- and interobserver reproducibility of rest and cold pressor test-stimulated myocardial blood flow with ${ }^{13} \mathrm{~N}$-ammonia and PET. Eur J Nucl Med Mol Imaging. 2007;34: 1178-1188.

59. Schindler TH, Facta AD, Prior JO, et al. PET-measured heterogeneity in longitudinal myocardial blood flow in response to sympathetic and pharmacologic stress as a non-invasive probe of epicardial vasomotor dysfunction. Eur J Nucl Med Mol Imaging. 2006;33:1140-1149.

60. Prior JO, Quinones MJ, Hernandez-Pampaloni M, et al. Coronary circulatory dysfunction in insulin resistance, impaired glucose tolerance, and type 2 diabetes mellitus. Circulation. 2005;111:2291-2298.

61. Schindler TH, Facta AD, Prior JO, et al. Improvement in coronary vascular dysfunction produced with euglycaemic control in patients with type 2 diabetes. Heart. 2007;93:345-349.

62. Quinones MJ, Hernandez-Pampaloni M, Schelbert H, et al. Coronary vasomotor abnormalities in insulin-resistant individuals. Ann Intern Med. 2004;140:700-708.

63. Morita K, Tsukamoto T, Naya M, et al. Smoking cessation normalizes coronary endothelial vasomotor response assessed with ${ }^{15} \mathrm{O}$-water and PET in healthy young smokers. J Nucl Med. 2006;47:1914-1920.

64. Lindner O, Vogt J, Kammeier A, et al. Effect of cardiac resynchronization therapy on global and regional oxygen consumption and myocardial blood flow in patients with non-ischaemic and ischaemic cardiomyopathy. Eur Heart J. 2005;26:70-76.

65. Wielepp P, Baller D, Gleichmann U, Pulawski E, Horstkotte D, Burchert W. Beneficial effects of atorvastatin on myocardial regions with initially low vasodilatory capacity at various stages of coronary artery disease. Eur J Nucl Med Mol Imaging. 2005;32:1371-1377.

66. Selwyn AP, Allan RM, L'Abbate A, et al. Relation between regional myocardial uptake of rubidium- 82 and perfusion: absolute reduction of cation uptake in ischemia. Am J Cardiol. 1982;50:112-121.

67. Anagnostopoulos C, Almonacid A, El Fakhri G, et al. Quantitative relationship between coronary vasodilator reserve assessed by ${ }^{82} \mathrm{Rb}$ PET imaging and coronary artery stenosis severity. Eur J Nucl Med Mol Imaging. 2008;35:1593-1601.

68. Lortie M, Beanlands RS, Yoshinaga K, Klein R, Dasilva JN, DeKemp RA. Quantification of myocardial blood flow with ${ }^{82} \mathrm{Rb}$ dynamic PET imaging. Eur J Nucl Med Mol Imaging. 2007;34:1765-1774.

69. Yoshinaga $\mathrm{K}$, Chow BJ, Williams $\mathrm{K}$, et al. What is the prognostic value of myocardial perfusion imaging using rubidium-82 positron emission tomography? J Am Coll Cardiol. 2006;48:1029-1039.
70. Schenker MP, Dorbala S, Hong EC, et al. Interrelation of coronary calcification, myocardial ischemia, and outcomes in patients with intermediate likelihood of coronary artery disease: a combined positron emission tomography/computed tomography study. Circulation. 2008;117:1693-1700.

71. Siegrist PT, Husmann L, Knabenhans M, et al. ${ }^{13} \mathrm{~N}$-ammonia myocardial perfusion imaging with a PET/CT scanner: impact on clinical decision making and cost-effectiveness. Eur J Nucl Med Mol Imaging. 2008;35:889-895.

72. Higuchi T, Abletshauser C, Nekolla SG, Schwaiger M, Bengel FM. Effect of the angiotensin receptor blocker Valsartan on coronary microvascular flow reserve in moderately hypertensive patients with stable coronary artery disease. Microcirculation. 2007;14:805-812.

73. Camici PG. From microcirculation to cardiac event: protection with Preterax. J Hypertens. 2008;26(suppl 2):S8-S10.

74. Naoumova RP, Kindler H, Leccisotti L, et al. Pioglitazone improves myocardial blood flow and glucose utilization in nondiabetic patients with combined hyperlipidemia: a randomized, double-blind, placebo-controlled study. J Am Coll Cardiol. 2007;50:2051-2058.

75. Neglia D, De Maria R, Masi S, et al. Effects of long-term treatment with carvedilol on myocardial blood flow in idiopathic dilated cardiomyopathy. Heart. 2007;93:808-813

76. Camici P, Chiriatti G, Lorenzoni R, et al. Coronary vasodilation is impaired in both hypertrophied and nonhypertrophied myocardium of patients with hypertrophic cardiomyopathy: a study with nitrogen-13 ammonia and positron emission tomography. J Am Coll Cardiol. 1991;17:879-886.

77. Olivotto I, Cecchi F, Gistri R, et al. Relevance of coronary microvascular flow impairment to long-term remodeling and systolic dysfunction in hypertrophic cardiomyopathy. J Am Coll Cardiol. 2006;47:1043-1048.

78. Cecchi F, Olivotto I, Gistri R, Lorenzoni R, Chiriatti G, Camici PG. Coronary microvascular dysfunction and prognosis in hypertrophic cardiomyopathy. $N$ Engl J Med. 2003;349:1027-1035.

79. Knaapen P, Germans T, Camici PG, et al. Determinants of coronary microvascular dysfunction in symptomatic hypertrophic cardiomyopathy. Am J Physiol Heart Circ Physiol. 2008;294:H986-H993.

80. Kaufmann PA, Rimoldi O, Gnecchi-Ruscone T, Bonser RS, Luscher TF, Camici PG. Systemic inhibition of nitric oxide synthase unmasks neural constraint of maximal myocardial blood flow in humans. Circulation. 2004;110:1431-1436.

81. Kaufmann PA, Rimoldi OE, Gnecchi-Ruscone T, Luscher TF, Camici PG. Systemic nitric oxide synthase inhibition improves coronary flow reserve to adenosine in patients with significant stenoses. Am J Physiol Heart Circ Physiol. 2007;293:H2178-H2182.

82. L'Abbate A, Camici P, Trivella MG, et al. Time-dependent response of coronary flow to prolonged adenosine infusion: doubling of peak reactive hyperaemic flow. Cardiovasc Res. 1981;15:282-286.

83. Heusch G, Baumgart D, Camici P, et al. $\alpha$-Adrenergic coronary vasoconstriction and myocardial ischemia in humans. Circulation. 2000;101:689-694.

84. Lorenzoni R, Rosen SD, Camici PG. Effect of alpha 1-adrenoceptor blockade on resting and hyperemic myocardial blood flow in normal humans. Am J Physiol. 1996;271:H1302-H1306 